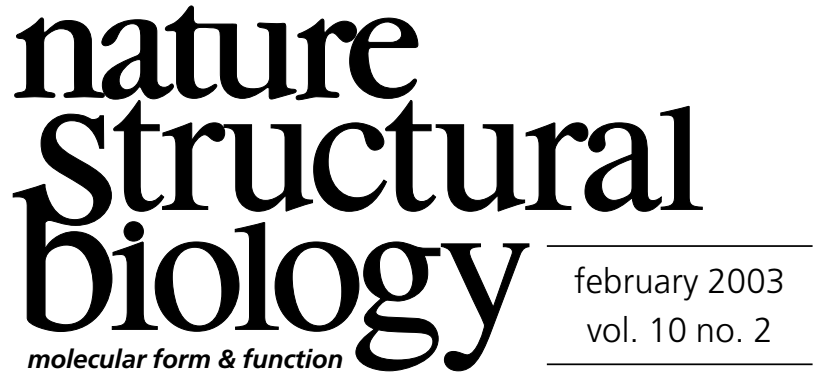

\title{
Recent changes at the journal
}

Regular readers of Nature Structural Biology will have noticed a significant change in the presentation of primary research papers recently. As of January 2003, all research papers are being published either as full-length 'Articles' or shorter 'Brief Communications'. To be precise, an Article can occupy four to ten printed pages of the journal (equating to a maximum of 5,000 words for the main text and Methods sections together), whereas a Brief Communication cannot exceed two printed pages $(\sim 1,000$ words) of the journal.

Why have we decided to do away with our 'Letters' section? In mid-1999, we broadened the scope of the journal to include functional studies that provide structural or mechanistic insight into biological processes (for details of our thoughts on the journal's scope, see Nat. Struct. Biol. 6, 495-496; 1999). Since that time, we have observed an increase in the number of papers that could not be easily accommodated in the Letter format. Some of these papers might report biochemical data in addition to a structure determination, for example, and extra space would be needed for the functional data. Other papers might report detailed biochemical or molecular biological studies and could require additional space to describe the results properly and discuss their implications. Both of these scenarios will be important in the journal's future evolution, and we believe that the Letter format has outlived its usefulness.

The Article format gives authors some leeway to explain their findings in the necessary detail, which could potentially improve the accessibility of their papers. In addition, research papers published in the Article format will include the full titles of all cited papers; we feel this will be valuable to both readers and editors alike. Within the Article format we will have the flexibility to publish each paper at its optimal length, and the editors at
Nature Structural Biology will work with authors throughout the editing process to achieve this key goal.

Readers should also note that Nature Structural Biology, together with Nature and other journals published by the Nature Publishing Group, has now adopted a changed policy regarding copyright. The essence of this policy is that our authors will now own the copyright to their work published in the journal, and we require authors to grant us an exclusive license to publish their papers in all forms of media worldwide. This means that authors may reproduce their contribution (or extracts from it) in their own books without having to ask us for permission, for example, provided that an acknowledgment to the original publication is included. Authors may also reproduce their own papers for the purpose of course teaching without paying a fee.

Under the license agreement, the authors may post their papers on their own not-for-profit web site, as long as a link to the Nature Structural Biology web site is provided. Hosting papers in open archives would be considered a breach of the license, however, and is not permitted. Although it will not be practicable to alter the copyright agreements covering material already published in the journal, we are delighted to be able to extend this new policy retroactively to authors of all contributions published in Nature Structural Biology. Please consult the small print at http://npg.nature.com/authornews.

As Nature Structural Biology approaches its tenth birthday, we are planning further changes in the journal that will take effect during the year. Some are designed to enhance the appearance of the journal, and others will provide new services to our readers. As always, we welcome your feedback, which can be sent to the editor (e-mail:nsb@natureny.com). 